\title{
Dopoguerra. Gli italiani fra speranze e disillusioni (1945-1947)
}

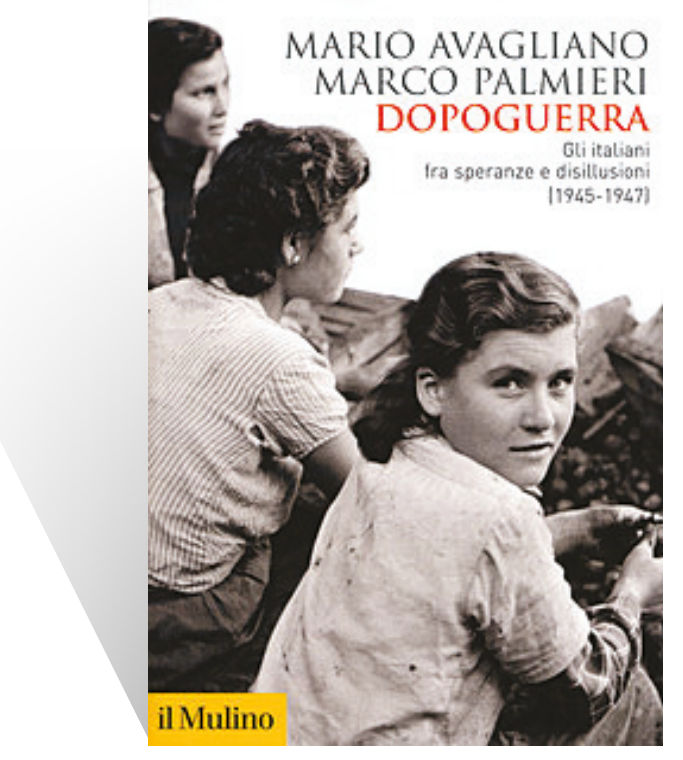

FICHA BIBLIOGRÁFICA

Mario Avagliano, Marco Palmieri, Dopoguerra. Gli italiani fra speranze e disillusioni (1945-1947), Bologna, il Mulino, 2019, Pp. 496, ISBN: 8815283714.

\section{Patrizia Gabrielli I Università di Siena}

CON QUESTO VOLUME SUlla STORIA DEL SECONDO DOPOGUERRA e della ricostruzione in Italia, Mario Avagliano e Marco Palmieri arricchiscono un progetto di ricerca che ha già visto con il Mulino tre pubblicazioni sulla seconda guerra mondiale e sull' Italia repubblicana (Vincere e vinceremo! Gli italiani al fronte 1940-1943 (2014); L'Italia di Salò 19431945 (2017); 1948. Gli italiani nell'anno della svolta (2018). Articolato in quattordici capitoli, per un totale di 496 pagine, il volume presenta una narrazione fluida e uno stile coinvolgente. Si tratta di una piacevole lettura che offre uno spaccato e più di un approfondimento su una fase storica complessa e di difficile gestione, le cui delicatezza e difficoltà sono egregiamente sintetizzate nell'espressione di Suso Cecchi D’Amico, sceneggiatrice di pregio: «Sta per scoppiare il dopoguerra». La guerra aveva prodotto distruzione, morte e gravi fratture, di tutto 
ciò molti testimoni ne avevano piena percezione. Riprendere «il corso normale» - osservava Andreina Zaninetti Libano, partigiana del Pd'A - avrebbe richiesto un paziente e tenace impegno, perché «non si risolve di botto una situazione come la nostra». Ed aveva ragione. Dati alla mano, gli Autori disegnano una panoramica sui problemi economici, politici, sociali che premono sugli italiani e sulle italiane, adulti e bambini.

Il volume è basato su una ricca e variegata gamma di fonti che vanno da un'aggiornata bibliografia ai documenti d'archivio, dalla documentazione raccolta presso gli Archivi di Stato, prodotta, dunque, dalle istituzioni, fino alle scritture della "gente comune" custodite dall'Archivio diaristico nazionale di Pieve Santo Stefano, anzi, a tale proposito possiamo affermare che alcune diariste, è il caso di Anna Marucelli e Rosalba Tradardi, già valorizzate in altre ricerche, divengono vere protagoniste di questa storia, quella che si svolge nel lungo dopoguerra Italiano. Meritano particolare menzione le lettere censurate che consentono di misurare non tanto o solo le condizioni di vita degli italiani, le loro assillanti preoccupazioni quotidiane quanto gli stati d'animo, i sentimenti provati. La censura della corrispondenza privata però invita a riflettere su i germi dell'autoritarismo ancora attivi ed operanti nel sistema italiano del post-fascismo. Integrano questo ventaglio di fonti i tanti riferimenti al cinema, alla musica, soprattutto a quella popolare, al teatro, agli eventi sportivi, alla letteratura che fanno di questa ricerca anche un prezioso strumento a fini didattici. Il ricco assortimento di documenti avrebbe richiesto un maggiore rigore filologico, una più attenta contestualizzazione, almeno la distinzione tra fonti edite e inedite. Questa disattenzione non inficia tuttavia la ricchezza della trattazione dedicata a un difficile e intenso biennio, quello che si snoda nel lungo dopoguerra, che vede l'Europa ridotta a «un continente selvaggio» e l'Italia a un cumulo di macerie, in senso letterale e metaforico. Di questa fase vengono messe in evidenza le tante luci e ombre restituendo al lettore un ampio quadro sulla storia del Paese nei suoi aspetti economici, politici, culturali e sociali. Ricostruire l'economia e le infrastrutture, sanare la disoccupazione, favorire il reinserimento dei reduci e dei prigionieri, fondare il nuovo ordinamento politico, le forme di governo nazionali e locali; sono questi i principali e gravosi compiti cui fare fronte. Un compito assai difficile se si tiene conto delle avversioni generate da quel surplus di violenza proprio delle guerre civili e nel biennio 1945-46 tensioni e rancori sono tutt'altro che sopiti, come confermano i diversi casi di violenza cui queste alcune pagine fanno riferimento.

Sulla base di statistiche e valutazioni di differente provenienza, viene offerta una panoramica sui problemi economici e sociali che assillano il paese e il quadro è affatto confortante. La ricostruzione è un processo che coinvolge l'economia, le infrastrutture, il lavoro, l'approvvigionamento, ovvero l'insufficiente e squilibrato regime alimentare degli italiani. Presto bisogna fare fronte al reinserimento dei reduci e dei prigionieri, circa un milione e mezzo di uomini «miracolosamente sopravvissuti» (p. 195) tornano a casa. L'Italia diviene un crocevia per il passaggio di molti profughi, soprattutto ebrei sopravvissuti alla Shoah. Da qui la necessaria organizzazione di strutture e l'avvio di adeguate politiche di accoglienza e di rinserimento. Questioni che si ripropongono, dopo il trattato di pace, con l'arrivo dei profughi giuliano dalmati. 
Ci sono poi le questioni politiche, il Paese deve assolvere il grave compito di fondare il nuovo ordinamento, le forme di governo nazionali e locali in un quadro frammentato, come conferma la «selva di partiti e movimenti» (p. 77) che operano in quel contesto; le violenze, «la sete di vendetta» (p. 53) strascico della guerra civile, o meglio, «scorie di una velenosa guerra civile» (p. 11). Tensioni e rancori si manifestano mentre il sistema giudiziario sembra non dare adeguate risposte e la amnistia, anche a causa della mancata applicazione delle disposizioni, lascia molto malcontento.

Sono pure anni di speranze. Gli Autori riservano un particolare spazio a quella straordinaria gioia di vivere che esplode in Italia di cui danno conferma la musica e il ballo, che si attestano quale antidoto al dolore, quel dolore interpretato e restituito in forma sublime nel dramma di Eduardo De Filippo in Napoli milionaria!: «'a guerra nun è fernuta» - afferma il reduce Gennaro Jovine protagonista dell'opera - S'ha d'aspettà. Ha da passa' 'a nuttata. Parole e stati d'animo condivisi tanto da creare un clima di densa e sincera commozione tra il pubblico che assisteva al San Carlo di Napoli alla prima. Eduardo De Filippo con il teatro sono un'importante tessera del fermento culturale di quegli anni che vede una ripresa del cinema, della produzione letteraria, delle riviste culturali.

La Repubblica nasce dalla consultazione referendaria, preceduta da una campagna elettorale di cui si delineano i caratteri, compresa la inedita presenza femminile, così come sono illustrate le differenti posizioni e il dibattito interno e tra i partiti politici, e tra questi e la Corona che oppone resistenza.

Sarebbe un errore pensare a un totale immobilismo sia per quanto concerne il quadro economico sia politico. Gli Autori ampiamente dimostrano come il Paese lentamente riprenda. Si lanciano sul mercato i primi beni di consumo, la Vespa, la Lambretta, la Ferrari simbolo della ripresa e della creatività italiana, mentre le case di moda confezionano modelli apprezzati oltre frontiera; nasce l'Algida con i suoi gelati confezionati; fa la sua comparsa nella vita di molti italiani "la schedina" e in tanti sperano in una bella vincita che possa riassestare le finanze familiari.

Restano aperti vecchi problemi, tanti i «dilemmi della Ricostruzione» (p. 333) ai quali sono dedicate tra l'altro le pagine sulla tanto discussa amnistia, sulla quale è offerta un'attenta disamina; si fa luce sui limiti e soprattutto sulla mancata applicazione della normativa paralizzata dalle mancanze e dalle inefficienze della burocrazia.

Invitano alla riflessione alcuni passaggi sulle manifestazioni contadine del dopoguerra e, in questo contesto, l'uccisione di Giuditta Levato, una contadina che partecipava alla simbolica occupazione delle terre nel Meridione, assurge a simbolo dell'arretratezza delle campagne, delle mancate riforme, «del divario tra ricchi e poveri», dell' intervento autoritario dello Stato.

Il 1947 è l'anno che conferisce all' Italia una nuova fisionomia politica. L'Assemblea Costituente e le relative commissioni, i leader e i gruppi dirigenti dei partiti lavorano fitto, intessono un dialogo, realizzano accordi, mediazioni, compromessi. Se nello scenario internazionale la guerra fredda è una realtà con effetti significativi sulle alleanze di governo, i partiti manifestano la volontà politica di collaborare in nome dell' interesse generale del Paese e alla fine del dicembre del 1947 la Costituzione della Repubblica italiana è un fatto compiuto. 
Merita sottolineare la scelta degli Autori di considerare aspetti ancora poco scandagliati dalla ricerca storica. È il caso non tanto o solo delle pagine sulla «caccia» (p. 53) alle fasciste e collaborazioniste, agli stupri di guerra, alla prostituzione, che chiamano in causa il segno di genere della violenza di guerra, quanto di quei passaggi capaci di illuminare fenomeni rimasti nascosti, tra i quali la difficoltà di ritrovare una normalità anche sul piano delle relazioni e degli affetti. Una figura simbolo in tal senso sembra incarnarsi nelle spose di guerra e nelle madri nubili. Belle le pagine dedicate ad altri spesso dimenticati protagonisti della storia: $i$ bambini anch'essi colpiti dagli strascichi di una guerra totale che non li ha risparmiati. Se a Napoli «fin dal 1944 le strade sono affollate di ragazzini con i vestiti a brandelli, inginocchiati davanti alle cassette di legno, sulle quali battone delle spazzole urlando "Sciuscià, sciuscià"» (p. 145), storpiatura dell' inglese shoes-shine, lustrare le scarpe, a Roma la delinquenza minorile si raccoglie alla Stazione Termini, nelle via adiacenti "più frequentate dai soldati alleati, come Via Nazionale, Piazza Esedra, Via Veneto e Via del Tritone tentando di offrire i loro servizi o vendere sigarette e preservativi» (p.146). Un grave fenomeno di marginalità dell' infanzia sul quale ci ha consegnato una vivida testimonianza il cinema del neorealismo. A Roma nel 1945 Vittorio De Sica gira Sciuscià, il film che esce nel 1946 e otterrà l'Oscar come miglior film straniero. 